\title{
Emotion Dysregulation and Inflammation in African-American Women with Type 2 Diabetes
}

\author{
Abigail Powers, ${ }^{1}$ Vasiliki Michopoulos, ${ }^{1}$ Karen Conneely, ${ }^{2}$ Rachel Gluck, ${ }^{1}$ \\ Hayley Dixon, ${ }^{1}$ Joseph Wilson, ${ }^{1}$ Tanja Jovanovic, ${ }^{1}$ Thaddeus W. W. Pace, ${ }^{3}$ \\ Guillermo E. Umpierrez, ${ }^{4}$ Kerry J. Ressler, ${ }^{1,5}$ Bekh Bradley, ${ }^{1,6}$ and Charles F. Gillespie ${ }^{1}$ \\ ${ }^{1}$ Department of Psychiatry and Behavioral Sciences, Emory University School of Medicine, Jesse Hill Jr. Drive, Atlanta, GA 30306, USA \\ ${ }^{2}$ Department of Human Genetics, Emory University School of Medicine, Atlanta, GA, USA \\ ${ }^{3}$ College of Nursing \& College of Medicine (Psychiatry), University of Arizona, Tucson, AZ, USA \\ ${ }^{4}$ Division of Endocrinology, Department of Medicine, Emory University School of Medicine, Atlanta, GA, USA \\ ${ }^{5}$ Center for Depression, Anxiety, and Stress Research, Harvard University, Cambridge, MA, USA \\ ${ }^{6}$ Atlanta VA Medical Center, Atlanta, GA, USA
}

Correspondence should be addressed to Charles F. Gillespie; cgilles@emory.edu

Received 10 February 2016; Revised 3 May 2016; Accepted 5 June 2016

Academic Editor: Stefan Kloiber

Copyright (C) 2016 Abigail Powers et al. This is an open access article distributed under the Creative Commons Attribution License, which permits unrestricted use, distribution, and reproduction in any medium, provided the original work is properly cited.

C-reactive protein (CRP), a marker of systemic inflammation, has been associated with major depressive disorder (MDD) and posttraumatic stress disorder (PTSD). Emotion dysregulation is a transdiagnostic risk factor for many psychological disorders associated with chronic inflammatory state. The objective of this study was to determine whether inflammation is associated with emotion dysregulation in women with type 2 diabetes mellitus (T2DM). We examined associations between trauma exposure, MDD, PTSD, emotion dysregulation, and CRP among 40 African-American women with T2DM recruited from an urban hospital. Emotion dysregulation was measured using the Difficulties in Emotion Regulation Scale. PTSD and MDD were measured with structured clinical interviews. Child abuse and lifetime trauma load were also assessed. Analyses showed that both emotion dysregulation and current MDD were significantly associated with higher levels of CRP $(p<0.01)$. Current PTSD was not significantly related to CRP. In a regression model, emotion dysregulation was significantly associated with higher CRP $(p<0.001)$ independent of body mass index, trauma exposure, and MDD diagnosis. These findings suggest that emotion dysregulation may be an important risk factor for chronic inflammation beyond already known risk factors among women with T2DM, though a causal relationship cannot be determined from this study.

\section{Introduction}

Major depressive disorder (MDD) and posttraumatic stress disorder (PTSD) have been associated with higher incidence of cardiovascular disease [1-4] and diabetes [5, 6]. One mechanism that may contribute to the comorbidity between psychopathology and adverse health outcomes is chronic inflammation, whose role in atherosclerosis is well established $[7,8]$. C-reactive protein (CRP) is a marker of systemic inflammation and provides a useful indicator of chronic inflammation. Increased circulating concentrations of CRP are associated with MDD [7, 9-11]. Increased circulating concentrations of CRP have also been described in PTSD [12-14]; however, the relationship is not as clear as other data are equivocal [15-17]. Because inflammation may be a key factor in understanding the significant comorbidity between psychiatric disorders and physical diseases, establishing other psychological factors that help explain the relationship between inflammation and psychiatric disorders and how problems with inflammation may develop is critical.

Ample evidence indicates that early life stress is associated with chronic inflammation in adulthood $[18,19]$, and this may be particularly true among African Americans [20]. For example, a recent meta-analysis on the role of childhood 
maltreatment (i.e., childhood sexual, physical, and emotional abuse and physical and emotional neglect) in inflammation in adulthood demonstrated a significant positive association between exposure to childhood trauma and higher circulating concentrations of CRP [21]. Childhood maltreatment exposure may be particularly detrimental to the immune system via problematic alterations in the function of the hypothalamic pituitary adrenal (HPA) axis, a major component of the body's stress response system and a system that both modulates and is modulated by inflammatory processes $[22,23]$. Based on this evidence, some posit that chronic inflammation may be one mediator in the relationship between early trauma exposure and the later development of psychiatric disorders [21]. It is important to note that the results of the meta-analysis on childhood trauma and inflammation suggested a relatively subtle (although significant) effect of trauma exposure on inflammatory activation suggesting that there may be other important components to this pathway that should be considered.

Emotion dysregulation, or deficits in awareness and management of intense negative emotional states, is a transdiagnostic risk factor for the development and maintenance of many psychological disorders, including depression and PTSD [23, 24], and may be another psychological factor that impacts chronic inflammation. Importantly, emotional development begins early in life and developmental research has shown a strong relationship between exposure to childhood maltreatment and the development of emotion regulation deficits in adolescence and adulthood [25-27]. This may occur in part because emotion regulation strategies are learned by caregivers and when family environments are harmful or unsupportive, children are less likely to be exposed to appropriate regulatory behavior and more likely to experience emotional invalidation where emotional expression is ignored, rejected, or punished [28]. Therefore, emotion dysregulation may be an important mechanism by which early life adversity confers lifetime risk for psychological disorders $[25,26]$. Based on the strong link between childhood maltreatment and emotion dysregulation, it is very possible that emotion dysregulation may also relate to chronic inflammation.

Recent research in fact suggests that emotion functioning is related to inflammation. A longitudinal study examining emotional functioning in 7-year-old children showed that inappropriate self-regulation and distress proneness during childhood predicted higher circulating concentrations of CRP in adulthood [29]. Other cross-sectional research on adults looked specifically at emotion regulation and showed that reappraisal (an adaptive emotion regulation skill) was associated with lower circulating concentrations of CRP while suppression (a maladaptive emotion regulation skill) was associated with elevated circulating concentrations of CRP [30]. Taken together, these studies indicate that emotion dysregulation could be important in predicting chronic inflammation, although no studies to our knowledge have examined the specific association between emotion dysregulation and circulating concentrations of CRP among traumatized adults with high rates of depression and PTSD.
One population at particular risk for elevated systemic inflammation is individuals with type 2 diabetes mellitus (T2DM). Multiple recent longitudinal studies show that elevated circulating concentrations of CRP put individuals at greater risk of developing T2DM [31, 32]. Understanding psychological factors that may be associated with increased inflammation levels in individuals with T2DM could be useful when considering how to improve treatments. Therefore, the goal of the present study was to examine the differential associations between trauma exposure, emotion dysregulation, current MDD, current PTSD, and CRP concentrations in a highly traumatized urban, minority sample of women with T2DM. We hypothesized that emotion dysregulation would be associated with higher concentrations of CRP independent of the effects of trauma exposure and current psychiatric diagnoses (i.e., PTSD and MDD).

\section{Material and Methods}

2.1. Procedure. Participants were drawn from astudy of risk factors for the development of PTSD in a low socioeconomic, urban minority population. Participants were recruited from waiting rooms in the diabetic, gynecology, and primary care medical clinics at a publically funded hospital in Atlanta, Georgia. We did not narrow recruitment to specific criteria but approachedany individual in the waiting room. To be eligible for participation, subjects had to be between the ages of 18 and 65 and able to give informed consent (see [33] for full details regarding study procedures). The investigation was carried out in accordance with the latest version of the Declaration of Helsinki and informed consent of the participants was obtained after the nature of the procedures had been fully explained. After signing the informed consent approved by the Emory Institutional Review Board and the Research Oversight Committee of Grady Memorial Hospital, an interview was administered with questionnaires regarding trauma history and psychological variables. Trained research assistants administered this interview (approximately 45-75 minutes).

A subgroup of female participants with T2DM was chosen for a separate associated study. These women returned to participate in structured clinical interviews and phlebotomy. Exclusion criteria included current bipolar or psychotic disorder diagnosis, alcohol or substance dependence, treatment for an autoimmune disorder, treatment with nonsteroidal anti-inflammatory, glucocorticoid, or anticonvulsant, and current treatment with an antipsychotic, benzodiazepine, or antidepressant. On the morning of the interview, height and weight were measured for calculation of body mass index (BMI) and fasting blood samples were collected for later batch assessment of CRP concentrations (approximately 2 weeks after initial assessment).

2.2. Participants. The current study included 40 diabetic African-American women with a mean age of 51.88 years $(\mathrm{SD}=7.57$ years, range $=32-65)$. Education levels were as follows: $18.8 \%$ reported less than high school education, $21.9 \%$ reported having a high school diploma or GED, 37.5\% 
reported having some college or technical school education, and $21.9 \%$ reported graduating from technical school or college. Only $25.0 \%$ of participants were employed and $84.4 \%$ had a household monthly income of $\$ 1999$ or less.

\subsection{Measures}

2.3.1. Difficulties in Emotion Regulation Scale (DERS). The DERS is a 36-item psychometrically validated [34] selfreport measure of emotion regulation difficulties. It measures several aspects of emotion regulation, including awareness and understanding of one's emotions, acceptance of negative emotions, the ability to successfully engage in goal-directed behavior and control impulsive behavior when experiencing negative emotions, and the ability to use situationally appropriate emotion regulation strategies. For the present study the overall scale, as well as six subscales, of emotion regulation was examined. The internal consistency of the DERS total scale was high $(\alpha=0.92)$.

2.3.2. Traumatic Events Inventory (TEI). The TEI is a 14-item screening instrument for lifetime history of traumatic events. It was administered to detail frequency and type of trauma(s) experienced; consistent with prior research [33], total level of trauma exposure was measured by a sum score reflecting the total frequency of different types of trauma (e.g., car accident, sexual assault) a participant had been exposed to over the course of their life. For this study, the TEI was used to measure frequency of lifetime trauma exposure. The number of traumatic experiences reflects the total number of different types of events that an individual experienced or witnessed. In order to separate lifetime trauma exposure and childhood abuse, we excluded the childhood abuse items from the TEI (i.e., exposure to sexual abuse, physical abuse, and emotional abuse).

2.3.3. Childhood Trauma Questionnaire (CTQ). The CTQ [35] is a 25-item, reliable, and valid self-report instrument assessing sexual, physical, emotional abuse, and neglect in childhood ( $\alpha=0.92$ in current study). Bernstein and Fink [36] established scores for none, mild, moderate, and severe for each type of abuse. For descriptive purposes, the data from the CTQ were used to create a categorical variable to account for the presence or absence of moderate-to-severe reported exposure to emotional (score $\geq 13$ ), physical (score $\geq 10$ ), and sexual (score $\geq 8$ ) abuse in childhood $(0=$ none or mild abuse; $1=$ the presence of moderate or severe abuse scores for at least one of the three types of abuse). A continuous measure of overall severity of childhood abuse exposure was also calculated and used as the measure of child abuse severity in all analyses.

2.3.4. Clinician-Administered PTSD Scale (CAPS). The CAPS is an interviewer-administered psychometrically validated diagnostic instrument measuring PTSD [37, 38]. It includes items that rate social and occupational functioning, global PTSD symptom severity, and the validity of participant's responses. The CAPS assesses current PTSD and was used to determine presence/absence of a PTSD diagnosis.

2.3.5. MINI International Neuropsychiatric Interview (MINI). MINI [39] is a structured diagnostic interview that assesses mood, anxiety, substance use, and psychotic disorders based on DSM-IV-TR criteria. MINI has shown good reliability and validity across different samples [39, 40]. For the present study, only the current major depression section was used to assess presence/absence of current MDD.

2.3.6. Body Mass Index (BMI). Body mass index is calculated as BMI $=$ body mass $(\mathrm{kg}) /(\text { height }(\mathrm{m}))^{2}$. Mean BMI for this sample was $36.53(\mathrm{SD}=7.30$, range $=21-53.80)$.

2.3.7. Highly Sensitive CRP. Serum samples were stored at $-80^{\circ} \mathrm{C}$ until the time of highly sensitive CRP (hsCRP) assay. Serum hsCRP concentrations were determined using an immunoturbidimetric assay from Sekisui Diagnostics (Lexington, MA) on the Beckman AU480 chemistry analyzer, with an interassay coefficient of variation (CV) of 5.2\% and an intra-assay $\mathrm{CV}$ of $3.1 \%$. Individuals with circulating concentrations of hsCRP $>20 \mathrm{mg} / \mathrm{L}$ were excluded from analysis because hsCRP $>20 \mathrm{mg} / \mathrm{L}$ suggests the presence of an active infection or other illnesses that could seriously confound study findings (two individuals were excluded from analyses (hsCRP $=26.25$ and 61.69)). Circulating concentrations of hsCRP averaged $5.70 \mathrm{mg} / \mathrm{L}(\mathrm{SD}=4.81$, range $=0.23-18.43)$.

2.3.8. Glucose. Plasma samples of glucose were stored at $-80^{\circ} \mathrm{C}$ until the time of assay. Glucose is measured by enzymatic methods on the Beckman AU480 using reagents from Beckman Coulter (Fullerton, CA). Average glucose level for this sample was $125.05(\mathrm{SD}=38.31$, range $=35.70-208.15)$.

2.3.9. Hemoglobin Alc (HbAlc). Whole blood samples were stored at $-80^{\circ} \mathrm{C}$ until the time of assay. HbAlc was measured using high performance liquid chromatography by ARUP laboratories (Salt Lake City, Utah). Average HbAlc for this sample was $7.61(\mathrm{SD}=1.71$, range $=4.90-13.60)$.

2.4. Data Analysis. The overall analytic approach was to examine the predictive utility of trauma, current PTSD, current MDD, and emotion dysregulation on hsCRP concentrations. We first examined the distributions of all key predictor variables. hsCRP concentrations, trauma exposure, and emotion dysregulation variables were positively skewed. However, the level of skewness (range: -0.32-1.96) in this sample fell within acceptable parameters for the sample size on all variables except child abuse severity [41] and the difficulty with emotion regulation strategies dimension of emotion dysregulation. Each had one outlier which affected the level of kurtosis, but results remained the same with or without these outliers included and so they remained in the models presented. Descriptive statistics of the variables of interest were computed (Table 1). Differences in trauma level, emotion dysregulation, and BMI were also examined by 
TABLE 1: Descriptive characteristics of variables of interest.

\begin{tabular}{|c|c|c|c|c|c|}
\hline & \multirow{4}{*}{$\begin{array}{c}\text { Overall sample } \\
\qquad N=40 \\
\text { Mean (SD, range) }\end{array}$} & \multicolumn{2}{|c|}{ Current depression } & \multicolumn{2}{|c|}{ Current PTSD } \\
\hline & & 0 & 1 & 0 & 1 \\
\hline & & $N=27$ & $N=13$ & $N=27$ & $N=13$ \\
\hline & & Mean (SD) & Mean (SD) & Mean (SD) & Mean (SD) \\
\hline Overall trauma load (excluding child abuse) & $4.31(2.27,1-12)$ & $4.52(2.38)$ & $3.86(2.02)$ & $3.97(2.00)$ & $5.00(2.70)$ \\
\hline Child abuse severity & $37.81(16.11,25-100)$ & $33.48(10.59)$ & $46.81(21.68)^{* *}$ & $31.37(7.65)$ & $51.19(20.71)^{* * *}$ \\
\hline Emotion dysregulation total & $72.75(24.81,39-145)$ & $64.78(19.56)$ & $89.31(27.06)^{* *}$ & $67.22(22.26)$ & $84.23(26.76)^{*}$ \\
\hline Nonacceptance of emotions & $12.10(5.48,6-27)$ & $10.67(4.69)$ & $15.08(5.98)^{*}$ & $11.26(5.35)$ & $13.85(5.54)$ \\
\hline Difficulty with goal-directed behavior & $11.52(5.00,5-25)$ & $9.48(2.89)$ & $15.77(5.88)^{* * *}$ & $9.93(3.11)$ & $14.85(6.54)^{* *}$ \\
\hline Difficulty controlling impulses & $11.20(4.865,6-21)$ & $9.48(2.89)$ & $13.31(4.92)$ & $10.52(5.09)$ & $12.62(4.17)$ \\
\hline Lack of awareness of emotions & $13.40(4.868,6-21)$ & $12.70(4.18)$ & $14.85(4.43)$ & $12.93(4.46)$ & $14.38(4.01)$ \\
\hline Lack of emotion regulation strategies & $14.48(5.99,8-36)$ & $12.63(4.60)$ & $18.31(6.86)^{* *}$ & $13.11(4.99)$ & $17.31(7.05)^{*}$ \\
\hline Lack of clarity of emotional experience & $10.05(4.47,5-23)$ & $9.11(4.44)$ & $12.00(4.02)$ & $9.48(4.45)$ & $11.23(4.46)$ \\
\hline Body mass index & $36.53(7.30,21-53.80)$ & $35.15(6.65)$ & $39.35(7.16)$ & $36.17(7.31)$ & $37.30(7.52)$ \\
\hline
\end{tabular}

Differences between groups were tested using one-way analysis of variance: ${ }^{*} p \leq 0.05 ;{ }^{* *} p \leq 0.01$; and ${ }^{* * *} p \leq 0.001$.

Note: trauma load was measured using TEI; child abuse severity was measured using CTQ; emotion dysregulation was measured using DERS; current PTSD was measured using CAPS; and current depressive episode was measured using MINI.

both MDD and PTSD diagnoses using analysis of variance (Table 1). For variables of interest, bivariate correlations (continuous variables) and a univariate analysis of variance (categorical variables) were also computed to determine associations with hsCRP in this sample. Then, based on the results of the correlational analyses, a series of linear regression models was fit to examine the unique predictive value of child abuse, current MDD, and emotion dysregulation on hsCRP. Potential covariates for regression analysis were first identified based on previous research suggesting associations with circulating concentrations of hsCRP: age, income, BMI, hemoglobin A1C, and baseline blood glucose level [9, 4245]. Due to the small sample size and risk for low power in detecting significant effects, associations between hsCRP and these potential covariates were first assessed to determine if their inclusion in the regression model was warranted. No significant differences emerged between any of the variables and hsCRP except for BMI $(r=0.40, p=0.01)$ and therefore only BMI was included as a covariate in the regression analysis. All analyses were conducted with SPSS 23.0 software package (twelve primary analyses were conducted resulting in a Bonferroni correction value of $p<0.004$ ).

\section{Results}

This was a highly traumatized sample, with all participants reporting the experience of at least one type of trauma in their lifetime (excluding child abuse; $\mathrm{M}=4.31, \mathrm{SD}=2.28$ ). Many participants were also exposed to childhood abuse (i.e. sexual, physical, or emotional abuse), with $35.0 \%$ of the sample reporting exposure to moderate-to-severe child abuse. Rates of depression and PTSD were also high in this sample, with $32.5 \%(n=13)$ meeting criteria for current MDD and $32.5 \%$ $(n=13)$ meeting criteria for current PTSD. The majority of those individuals that met diagnostic criteria for at least one diagnosis in fact met diagnostic criteria for both MDD and PTSD (22.5\% of overall sample, $n=9$ ). As shown in Table 1, individuals with depression and PTSD were more likely to report higher levels of child abuse $(p<0.01$ and $p<0.001$, resp. $)$ and emotion dysregulation $(p<0.01$ and $p<0.05$, resp.). Rates of other trauma exposure and BMI were not significantly different across groups.

To determine the extent of association between trauma exposure, emotion dysregulation, and hsCRP, we first calculated Pearson correlation coefficients among our variables of interest (see Table 2). BMI was also included in these analyses to evaluate independent associations with the variables of interest. Overall emotion dysregulation was significantly positively correlated with hsCRP concentrations $(p<0.001)$. When looking at the six dimensions of emotion dysregulation, all six were significantly positively correlated with difficulty controlling impulses and lack of emotion regulation strategies showing the strongest correlations $(p<0.001)$. All significant associations except for lack of awareness of emotions would survive correction for multiple comparisons. BMI ( $p=0.01)$ was also positively correlated with hsCRP. The association between child abuse severity and hsCRP trended toward significance $(p=0.067)$. However, overall trauma exposure (excluding child abuse) was not significantly correlated with hsCRP. Regarding associations between trauma exposure and emotion dysregulation, child abuse severity was associated with overall emotion dysregulation $(p<0.05)$ as well as nonacceptance of emotions $(p=0.01)$ and difficulty with goal-directed behavior in the presence of strong emotions $(p=0.05)$. Overall trauma exposure (excluding child abuse) was not associated with emotion dysregulation. However, child abuse severity and overall trauma exposure (excluding abuse) were significantly correlated with each other $(p<0.05)$. Regarding BMI, only one emotion dysregulation dimension-lack of awareness of emotions-was significantly associated with BMI $(p<0.05)$. Neither trauma exposure variable was significantly associated with BMI.

Univariate analysis of variance was then used to examine mean differences in hsCRP by PTSD and MDD diagnosis. As shown in Figure 1, univariate analysis of variance results showed that there was a significant increase in mean hsCRP 
TABle 2: Bivariate Pearson's correlations $(r)$ between variables of interest.

\begin{tabular}{|c|c|c|c|c|c|c|c|c|c|c|c|}
\hline & 1 & 2 & 3 & 4 & 5 & 6 & 7 & 8 & 9 & 10 & 11 \\
\hline Emotion dysregulation total & - & & & & & & & & & & \\
\hline Nonacceptance of emotions & $0.82^{* * *}$ & - & & & & & & & & & \\
\hline Difficulty with goal-directed behavior & $0.82^{* * *}$ & $0.56^{* * *}$ & - & & & & & & & & \\
\hline Difficulty controlling impulses & $0.80^{* * *}$ & $0.58^{* * *}$ & $0.58^{* * *}$ & - & & & & & & & \\
\hline Lack of awareness of emotions & $0.65^{* * *}$ & $0.33^{*}$ & $0.47^{* * *}$ & $0.44^{* *}$ & - & & & & & & \\
\hline Lack of emotion regulation strategies & $0.93^{* * *}$ & $0.76^{* * *}$ & $0.74^{* * *}$ & $0.65^{* * *}$ & $0.55^{* * *}$ & - & & & & & \\
\hline Lack of clarity of emotional experience & $0.89^{* * *}$ & $0.69^{* * *}$ & $0.65^{* * *}$ & $0.68^{* * *}$ & $0.51^{* * *}$ & $0.83^{* * *}$ & - & & & & \\
\hline Other trauma exposure & -0.02 & -0.01 & 0.07 & 0.14 & -0.11 & -0.04 & -0.18 & - & & & \\
\hline Child abuse severity & $0.32^{*}$ & $0.40^{* *}$ & $0.31^{*}$ & 0.20 & 0.15 & 0.23 & 0.27 & $0.38^{*}$ & - & & \\
\hline hsCRP & $0.73^{* * *}$ & $0.60^{* * *}$ & $0.67^{* * *}$ & $0.55^{* * *}$ & $0.44^{* *}$ & $0.73^{* * *}$ & $0.56^{* * *}$ & 0.14 & $0.29^{\dagger}$ & - & \\
\hline Body mass index & 0.21 & 0.14 & 0.14 & 0.06 & $0.32^{*}$ & 0.24 & 0.14 & -0.04 & 0.13 & $0.40^{* *}$ & - \\
\hline
\end{tabular}

${ }^{*} p \leq 0.05 ;{ }^{* *} p \leq 0.01 ;{ }^{* * *} p \leq 0.001 ;$ and ${ }^{\dagger} p=0.067$.

Note: emotion dysregulation was measured using DERS, child abuse severity was measured using CTQ, and trauma exposure was measured using TEI.

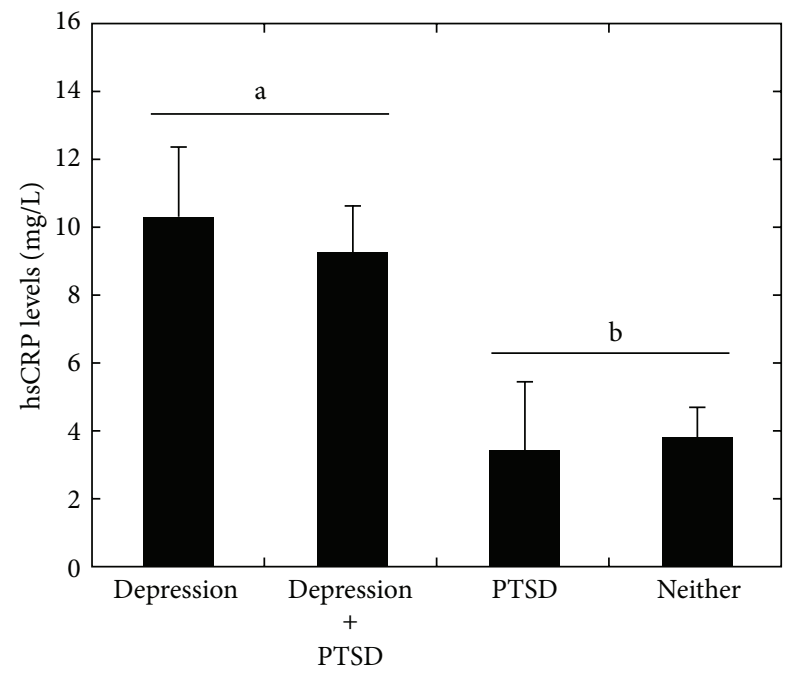

FIGURE 1: Univariate analysis of variance results predicting mean hsCRP levels by current depression and PTSD diagnoses. Significant main effect of depression denoted by letters $(p<0.001)$.

across current MDD diagnosis $(F=19.12, p<0.001)$ but not current PTSD diagnosis $(F=2.87, p=0.10)$. There was no significant interaction between MDD and PTSD diagnoses.

Next, a series of linear regression models was run to test the differential associations of child abuse, current MDD, and emotion dysregulation with hsCRP. BMI was entered in the first step of the regression model as a covariate. Then, child abuse severity was entered into the second step of the regression model. Next, current MDD was entered into the third step of the regression model. Finally, emotion dysregulation was entered into the fourth step of the regression model. As shown in Table 3, when BMI was included in step 1, higher BMI was significantly related to higher hsCRP levels $(p<0.05)$. When child abuse severity was included in step 2, it was not significant in predicting hsCRP. In step 3, when current MDD was entered into the regression model, MDD was significantly predictive of hsCRP ( $p<0.01$ ), accounting for $13 \%$ of the variance in
hsCRP independent of BMI and child abuse severity. When overall emotion dysregulation was included in the model in step 4, emotion dysregulation was significantly predictive of hsCRP ( $p<0.001$ ) above and beyond BMI, child abuse severity, and current MDD, surviving correction for multiple comparisons and accounting for $25 \%$ of unique variance in predicting higher hsCRP concentrations. Including PTSD in the model did not change the results; a model was also run with continuous measures of depression (MINI) and PTSD (CAPS) and significant results did not change.

\section{Discussion}

To our knowledge, the current study is the first to evaluate the differential relationship between emotion dysregulation, trauma exposure, psychiatric disorders, and circulating CRP concentrations in a traumatized sample of women. In support of our hypothesis, within this sample of African-American females with T2DM, we found that emotion dysregulation was significantly associated with higher peripheral concentrations of CRP. This result is consistent with recent research which has shown that emotional functioning in childhood predicts elevated CRP in adulthood [29] and expands previous findings to demonstrate that emotion dysregulation was significantly predictive of elevated CRP above and beyond already known risk factors, including BMI, trauma, and current MDD.

Although all dimensions of emotion dysregulation measured were significantly related to higher CRP, difficulty engaging in goal-directed behavior in the presence of strong emotions and a lack of strategies for managing strong negative emotions showed the strongest associations with elevated concentrations of CRP. These results indicate that heightened inflammation in those with high emotion dysregulation in the current study may be due to chronic activation of neuroendocrine and immune systems in response to chronic stress exposure as a result of a lack of effective strategies for managing negative emotions. Indeed, exposure to repeating and unrelenting psychosocial stressors results in the dysregulation of the feedback mechanisms that regulate the activity 
TABLE 3: Linear regression model predicting current hsCRP levels from trauma exposure, current MDD diagnosis, and overall emotion dysregulation symptoms.

\begin{tabular}{|c|c|c|c|c|c|c|c|}
\hline & $\beta$ & $t$ & $p$ & $R$ & $R^{2}$ change & $F$ change & $p$ change \\
\hline Step 1 & & & & 0.42 & 0.17 & 5.69 & $0.02^{*}$ \\
\hline BMI & 0.42 & 2.39 & $0.02^{*}$ & & & & \\
\hline Step 2 & & & & 0.49 & 0.07 & 2.34 & 0.14 \\
\hline BMI & 0.40 & 2.31 & $0.03^{*}$ & & & & \\
\hline Child abuse severity & 0.26 & 1.53 & 0.14 & & & & \\
\hline Step 3 & & & & 0.61 & 0.13 & 5.23 & $0.03^{*}$ \\
\hline BMI & 0.28 & 1.71 & 0.10 & & & & \\
\hline Child abuse severity & 0.08 & 0.47 & 0.64 & & & & \\
\hline Current MDD & 0.42 & 2.29 & $0.03^{*}$ & & & & \\
\hline Step 4 & & & & 0.79 & 0.25 & 16.39 & $<0.001$ \\
\hline BMI & 0.22 & 1.68 & 0.11 & & & & \\
\hline Child abuse severity & -0.05 & -0.32 & 0.75 & & & & \\
\hline Current MDD & 0.24 & 1.59 & 0.13 & & & & \\
\hline Emotion dysregulation & 0.58 & 4.05 & $<0.001$ & & & & \\
\hline
\end{tabular}

${ }^{*} p \leq 0.05$.

Note: child abuse severity was measured using CTQ; emotion dysregulation was measured using DERS; and current depressive episode was measured using MINI.

of the HPA axis and the inflammatory system [21, 22]. Under normal conditions, glucocorticoids are released in response to stressors that act to inhibit the activity of the immune system [46]. However, overactivation of the HPA axis in response to chronic stress exposure leads to dysfunction of the HPA axis and glucocorticoid responsiveness that leads to an attenuation of glucocorticoid-induced inhibition of the inflammatory response [46]. Thus, it is possible that an inability to manage negative emotions in response to psychosocial stressor exposure with adaptive strategies facilitates increased inflammation. Although the direction of causality cannot be established from an association study, improving emotion regulation strategies and individuals' ability to tolerate strong emotions and not act on them may be a useful area to focus on in treatment with individuals who have comorbid psychopathology and chronic inflammation. Future studies with a controlled experimental design are needed to assess whether improvements in emotional regulation can lead to reductions in inflammation. It is also critical that more research be conducted to dissect the associations between the various dimensions of emotion dysregulation and chronic inflammation to inform treatment decisions.

Consistent with previous research, we also found that current depression was associated with a proinflammatory state. Indeed, individuals with MDD have been shown in metaanalyses to have elevated CRP [7, 9-11], as well as increased levels of interleukin-6 (IL-6) and tumor necrosis factor- $\alpha$ $(\mathrm{TNF}-\alpha)[9,47]$. Causal pathways between depression and inflammation have been debated and much of the evidence has focused on the role of proinflammatory cytokines in the development of depressive symptoms and "sickness behavior" (e.g., anhedonia, sleep, and appetite changes [48-51]). However, not all individuals with depression show a heightened inflammatory state, and it is likely that in fact the positive association between depression and inflammation is a result of a complex and bidirectional process in which components of the central nervous system (e.g., HPA axis, autonomic nervous system) alter inflammation and depression which in turn impact one another [9]. Our finding that current depression is associated with higher CRP in females is helpful since some recent population-based studies found sex differences in associations between depression and CRP, such that the relationship was only found for men $[10,11]$. However, other studies looking specifically at women have found strong associations between MDD and higher levels of CRP in women $[52,53]$, and our finding indicates further support for this relationship among women. It will be important to continue evaluating sex differences in the association between inflammation and psychopathology as the type of population studied clearly impacts the interpretation and generalizability of the association.

While depression was significantly associated with CRP, current PTSD was not, which contradicts some previous findings [12-14]. However, the literature on this association is mixed, with studies also showing a negative association between PTSD and CRP [15] or no association at all [16, 17]. It is likely that the specificity of our sample (highly traumatized African-American females with T2DM) contributed to these results and therefore limits the generalizability of these results to other populations. There may be other pathways associated with the stress response system and emotion dysregulation that may be more relevant to PTSD than inflammation, such as enhanced glucocorticoid negative feedback of the HPA axis and heightened activity of the sympathetic nervous system [54]. Ongoing studies in our lab are beginning to examine how immune, neuroendocrine, and autonomic function all relate to PTSD and MDD in the context of acute stress.

Overall trauma exposure was also not related to circulating concentrations of CRP. It is possible that this is in part due to the fact that all women included in the 
study had been exposed to traumatic events (mean $=4.23$ types of events). However, although not significant in this sample, we did see a trend toward a positive association between child abuse and peripheral concentrations of CRP. Our small sample size made us underpowered to detect small effects, and it is likely that with more women included, this effect would be statistically significant. This result would fit with previous research finding a relationship between child abuse exposure and inflammation in adulthood [55]. Of note, emotion dysregulation and child abuse were significantly related, and based on the strong effect found between emotion dysregulation and CRP, it is possible that emotion dysregulation is the mechanism by which early trauma contributes to chronic inflammation, although this could not be explored in this cross-sectional study. More research is needed to determine potential risk pathways of trauma exposure and emotion regulation problems on the development of inflammation and should be examined in both traumatized and nontraumatized populations.

The current study has some limitations that should be kept in mind when interpreting the results. First, the study was cross-sectional in nature, which does not allow for the determination of causality. It is certainly possible that chronic inflammation leads to higher levels of emotion dysregulation, as some research has shown that inflammation can lead to mood and emotional changes [56]. However, the strong evidence that emotion dysregulation develops throughout childhood and is impacted by early life trauma [25-27, 57-59], along with initial evidence that the emotional functioning in childhood predicts adult CRP concentrations [29], provides support for emotion dysregulation as an underlying mechanism that might lead to chronic inflammation and later health problems such as cardiovascular disease and diabetes. In addition, emotion dysregulation was measured using a self-report questionnaire (DERS). This means that participants needed to have insight into their emotion regulation difficulties in order to report them. A lack of insight could have underestimated the severity of emotion dysregulation within this group of women. However, the DERS has shown good construct validity in its strong relationship with psychiatric symptoms and outcomes (e.g., PTSD symptoms, self-harm behavior [34, 60-62]). Recent research has also shown associations between the poor impulse control dimension of the DERS and lower activation of the rostral anterior cingulate cortex during an impulse control task, suggesting that this self-report measure of emotion dysregulation is related to already known neural mechanisms associated with adaptive emotion regulation, thus further supporting its validity. Another limitation is that the homogeneity of the study population means that the results may not be generalizable to other samples. This sample included all African-American women with T2DM and high levels of trauma exposure, thus exceeding the rates of psychiatric disorders generally found in community samples [33]. However, the homogeneous sample is a strength as African-American women with T2DM are clearly an at-risk sample that would benefit from improved interventions. Past research has shown increased inflammatory biomarkers in low-socioeconomic-status African-American communities
$[63,64]$ which is consistent with our sample of traumatized T2DM women, as overall peripheral concentrations of CRP in this sample were very high (mean $=5.70 \mathrm{mg} / \mathrm{L}, \mathrm{SD}=$ 4.80). Finally, the small sample size of this study means that we may not have had enough power to detect small, yet clinically important associations between the variables examined. This was evident in the association between child abuse severity and higher concentrations of CRP $(r=$ $0.29, p=0.067$ ) that was trending toward significance. This is the first study to examine the complex relationships between trauma, psychiatric disorders, self-reported emotion dysregulation, and CRP and therefore more research with larger sample sizes is needed to replicate these findings.

\section{Conclusions}

Within at-risk groups it may be particularly beneficial to target psychological treatment interventions that may also then impact inflammation. CRP is easily measured and is a significant predictor of risk for serious physical conditions like cardiovascular disease. Future research should examine how psychological interventions for depression and/or PTSD might differentially affect emotion dysregulation and CRP concentrations and whether changes in one might then influence the status of the other. There is already some evidence that reductions in chronic inflammation can result from treating depression [65] and that reducing inflammation decreases affective symptoms [66]. Emotion dysregulation may be an even more useful treatment target since it cuts across psychiatric disorders and can be implemented in the context of various treatment modalities (e.g., cognitivebehavioral, acceptance and commitment, and dialectical behavior therapies) and may be more easily integrated into nonpsychiatric settings (e.g., primary care and diabetes clinics) where brief skills training is more feasible than long-term psychotherapy.

\section{Disclosure}

The content is solely the responsibility of the authors and does not necessarily represent the official views of the National Institutes of Health. Additionally, the contents of this report do not represent the views of the Department of Veterans Affairs or the United States Government.

\section{Competing Interests}

There are no conflict of interests to disclose.

\section{Acknowledgments}

The authors would like to thank Allen W. Graham, Angelo Brown, Rebecca Roffman, and the entire Grady Trauma Project and Stress and Diabetes Study Project staff for their assistance in data collection and management. This work was primarily supported by the National Institute of Mental Health (MH071537 for K. J. Ressler, MH102890 for A. Powers, and MH099211 for C. F. Gillespie) and the National Institute 
of Child Health and Human Development (HD071982 for B. Bradley). Support also included Emory and Grady Memorial Hospital General Clinical Research Center, NIH National Centers for Research Resources (M01 RR00039).

\section{References}

[1] M.-L. Gander and R. von Känel, "Myocardial infarction and post-traumatic stress disorder: frequency, outcome, and atherosclerotic mechanisms," European Journal of Cardiovascular Prevention and Rehabilitation, vol. 13, no. 2, pp. 165-172, 2006.

[2] L. D. Kubzansky, K. C. Koenen, A. Spiro III, P. S. Vokonas, and D. Sparrow, "Prospective study of posttraumatic stress disorder symptoms and coronary heart disease in the Normative Aging Study," Archives of General Psychiatry, vol. 64, no. 1, pp. 109-116, 2007.

[3] D. L. Musselman, D. L. Evans, and C. B. Nemeroff, "The relationship of depression to cardiovascular disease: epidemiology, biology, and treatment," Archives of General Psychiatry, vol. 55, no. 7, pp. 580-592, 1998.

[4] J. Barth, M. Schumacher, and C. Herrmann-Lingen, "Depression as a risk factor for mortality in patients with coronary heart disease: a meta-analysis," Psychosomatic Medicine, vol. 66, no. 6, pp. 802-813, 2004.

[5] B. Mezuk, W. W. Eaton, S. Albrecht, and S. H. Golden, "Depression and type 2 diabetes over the lifespan," Diabetes Care, vol. 31, no. 12, pp. 2383-2390, 2008.

[6] R. D. Goodwin and J. R. Davidson, "Self-reported diabetes and posttraumatic stress disorder among adults in the community," Preventive Medicine, vol. 40, no. 5, pp. 570-574, 2005.

[7] H.-K. Kuo, C.-J. Yen, C.-H. Chang, C.-K. Kuo, J.-H. Chen, and F. Sorond, "Relation of C-reactive protein to stroke, cognitive disorders, and depression in the general population: systematic review and meta-analysis," The Lancet Neurology, vol. 4, no. 6, pp. 371-380, 2005.

[8] P. Libby, P. M. Ridker, and A. Maseri, "Inflammation and atherosclerosis," Circulation, vol. 105, no. 9, pp. 1135-1143, 2002.

[9] M. B. Howren, D. M. Lamkin, and J. Suls, "Associations of depression with c-reactive protein, IL-1, and IL-6: a metaanalysis," Psychosomatic Medicine, vol. 71, no. 2, pp. 171-186, 2009.

[10] D. E. Ford and T. P. Erlinger, "Depression and C-reactive protein in US adults: data from the Third National Health and Nutrition Examination Survey," Archives of Internal Medicine, vol. 164, no. 9, pp. 1010-1014, 2004.

[11] M. Danner, S. V. Kasl, J. L. Abramson, and V. Vaccarino, "Association between depression and elevated C-reactive protein," Psychosomatic Medicine, vol. 65, no. 3, pp. 347-356, 2003.

[12] V. Michopoulos, A. O. Rothbaum, T. Jovanovic et al., "Association of CRP genetic variation and CRP level with elevated PTSD symptoms and physiological responses in a civilian population with high levels of trauma," The American Journal of Psychiatry, vol. 172, no. 4, pp. 353-362, 2015.

[13] R. J. Miller, A. G. Sutherland, J. D. Hutchison, and D. A. Alexander, "C-reactive protein and interleukin 6 receptor in post-traumatic stress disorder: a pilot study," Cytokine, vol. 13, no. 4, pp. 253-255, 2001.

[14] C. Spitzer, S. Barnow, H. Völzke et al., "Association of posttraumatic stress disorder with low-grade elevation of C-reactive protein: evidence from the general population," Journal of Psychiatric Research, vol. 44, no. 1, pp. 15-21, 2010.
[15] H. P. Söndergaard, L.-O. Hansson, and T. Theorell, "The inflammatory markers C-reactive protein and serum amyloid a in refugees with and without posttraumatic stress disorder," Clinica Chimica Acta, vol. 342, no. 1-2, pp. 93-98, 2004.

[16] R. von Känel, U. Hepp, B. Kraemer et al., "Evidence for low-grade systemic proinflammatory activity in patients with posttraumatic stress disorder," Journal of Psychiatric Research, vol. 41, no. 9, pp. 744-752, 2007.

[17] E. C. McCanlies, S. K. Araia, P. N. Joseph et al., "C-reactive protein, interleukin-6, and posttraumatic stress disorder symptomology in urban police officers," Cytokine, vol. 55, no. 1, pp. 74-78, 2011.

[18] S. E. Taylor, B. J. Lehman, C. I. Kiefe, and T. E. Seeman, "Relationship of early life stress and psychological functioning to adult c-reactive protein in the coronary artery risk development in young adults study," Biological Psychiatry, vol. 60, no. 8, pp. 819-824, 2006

[19] A. Danese, C. M. Pariante, A. Caspi, A. Taylor, and R. Poulton, "Childhood maltreatment predicts adult inflammation in a lifecourse study," Proceedings of the National Academy of Sciences of the United States of America, vol. 104, no. 4, pp. 1319-1324, 2007.

[20] N. Slopen, T. T. Lewis, T. L. Gruenewald et al., "Early life adversity and inflammation in African Americans and whites in the midlife in the United States survey," Psychosomatic Medicine, vol. 72, no. 7, pp. 694-701, 2010.

[21] A. H. Miller, V. Maletic, and C. L. Raison, "Inflammation and its discontents: the role of cytokines in the pathophysiology of major depression," Biological Psychiatry, vol. 65, no. 9, pp. 732741, 2009.

[22] T. W. W. Pace, F. Hu, and A. H. Miller, "Cytokine-effects on glucocorticoid receptor function: relevance to glucocorticoid resistance and the pathophysiology and treatment of major depression," Brain, Behavior, and Immunity, vol. 21, no. 1, pp. 9-19, 2007.

[23] A. Aldao, S. Nolen-Hoeksema, and S. Schweizer, "Emotionregulation strategies across psychopathology: a meta-analytic review," Clinical Psychology Review, vol. 30, no. 2, pp. 217-237, 2010.

[24] B. Bradley, J. A. DeFife, C. Guarnaccia et al., "Emotion dysregulation and negative affect: association with psychiatric symptoms," Journal of Clinical Psychiatry, vol. 72, no. 5, pp. 685691, 2011.

[25] L. R. A. Alink, D. Cicchetti, J. Kim, and F. A. Rogosch, "Mediating and moderating processes in the relation between maltreatment and psychopathology: mother-child relationship quality and emotion regulation," Journal of Abnormal Child Psychology, vol. 37, no. 6, pp. 831-843, 2009.

[26] J. Kim and D. Cicchetti, "Longitudinal pathways linking child maltreatment, emotion regulation, peer relations, and psychopathology," Journal of Child Psychology and Psychiatry and Allied Disciplines, vol. 51, no. 6, pp. 706-716, 2010.

[27] K. Shipman, J. Zeman, S. Penza, and K. Champion, "Emotion management skills in sexually maltreated and nonmaltreated girls: a developmental psychopathology perspective," Development and Psychopathology, vol. 12, no. 1, pp. 47-62, 2000.

[28] M. Linehan, Cognitive-Behavioral Treatment of Borderline Personality Disorder, Guilford Press, New York, NY, USA, 1993.

[29] A. A. Appleton, S. L. Buka, M. C. McCormick et al., "Emotional functioning at age 7 years is associated with C-reactive protein in middle adulthood," Psychosomatic Medicine, vol. 73, no. 4, pp. 295-303, 2011. 
[30] A. A. Appleton, S. L. Buka, E. B. Loucks, S. E. Gilman, and L. D. Kubzansky, "Divergent associations of adaptive and maladaptive emotion regulation strategies with inflammation," Health Psychology, vol. 32, no. 7, pp. 748-756, 2013.

[31] A. D. Pradhan, J. E. Manson, N. Rifai, J. E. Buring, and P. M. Ridker, "C-reactive protein, interleukin 6, and risk of developing type 2 diabetes mellitus," The Journal of the American Medical Association, vol. 286, no. 3, pp. 327-334, 2001.

[32] D. J. Freeman, J. Norrie, M. J. Caslake et al., "C-reactive protein is an independent predictor of risk for the development of diabetes in the west of Scotland coronary prevention study," Diabetes, vol. 51, no. 5, pp. 1596-1600, 2002.

[33] C. F. Gillespie, B. Bradley, K. Mercer et al., "Trauma exposure and stress-related disorders in inner city primary care patients," General Hospital Psychiatry, vol. 31, no. 6, pp. 505-514, 2009.

[34] K. L. Gratz and L. Roemer, "Multidimensional assessment of emotion regulation and dysregulation: development, factor structure, and initial validation of the difficulties in emotion regulation scale," Journal of Psychopathology and Behavioral Assessment, vol. 26, no. 1, pp. 41-54, 2004.

[35] D. P. Bernstein, J. A. Stein, M. D. Newcomb et al., "Development and validation of a brief screening version of the Childhood Trauma Questionnaire," Child Abuse \& Neglect, vol. 27, no. 2, pp. 169-190, 2003.

[36] D. Bernstein and L. Fink, Manual for the Childhood Trauma Questionnaire, The Psychological Corporation, New York, NY, USA, 1998.

[37] D. D. Blake, F. W. Weathers, L. M. Nagy et al., "The development of a clinician-administered PTSD scale," Journal of Traumatic Stress, vol. 8, no. 1, pp. 75-90, 1995.

[38] D. D. Blake, F. W. Weathers, L. M. Nagy et al., A Clinician Rating Scale for Assessing Current and Lifetime PTSD: The CAPS1, 1990.

[39] D. V. Sheehan, Y. Lecrubier, K. H. Sheehan et al., "The validity of the Mini International Neuropsychiatric Interview (MINI) according to the SCID-P and its reliability," European Psychiatry, vol. 12, no. 5, pp. 232-241, 1997.

[40] Y. Lecrubier, D. V. Sheehan, E. Weiller et al., "The Mini International Neuropsychiatric Interview (MINI). A short diagnostic structured interview: reliability and validity according to the CIDI," European Psychiatry, vol. 12, no. 5, pp. 224-231, 1997.

[41] B. G. Tabachnick and L. S. Fidell, Using Multivariate Statistics, Pearson/Allyn \& Bacon, Boston, Mass, USA, 2001.

[42] D. E. Alley, T. E. Seeman, J. Ki Kim, A. Karlamangla, P. Hu, and E. M. Crimmins, "Socioeconomic status and C-reactive protein levels in the US population: NHANES IV," Brain, Behavior, and Immunity, vol. 20, no. 5, pp. 498-504, 2006.

[43] E. S. Ford, "Body mass index, diabetes, and C-reactive protein among U.S. Adults," Diabetes Care, vol. 22, no. 12, pp. 1971-1977, 1999.

[44] N. De Rekeneire, R. Peila, J. Ding et al., "Diabetes, hyperglycemia, and inflammation in older individuals: the health, aging and body composition study," Diabetes Care, vol. 29, no. 8, pp. 1902-1908, 2006.

[45] M. Kaefer, S. J. Piva, J. A. M. De Carvalho et al., "Association between ischemia modified albumin, inflammation and hyperglycemia in type 2 diabetes mellitus," Clinical Biochemistry, vol. 43, no. 4-5, pp. 450-454, 2010.

[46] P. H. Black, "Stress and the inflammatory response: a review of neurogenic inflammation," Brain, Behavior, and Immunity, vol. 16, no. 6, pp. 622-653, 2002.
[47] Y. Liu, R. C.-M. Ho, and A. Mak, "Interleukin (IL)-6, tumour necrosis factor alpha (TNF- $\alpha$ ) and soluble interleukin-2 receptors (sIL-2R) are elevated in patients with major depressive disorder: a meta-analysis and meta-regression," Journal of Affective Disorders, vol. 139, no. 3, pp. 230-239, 2012.

[48] E. B. Hekler, J. Rubenstein, E. J. Coups et al., "Inflammatory markers in acute myocardial infarction patients: preliminary evidence of a prospective association with depressive symptoms," Journal of Applied Biobehavioral Research, vol. 12, no. 2, pp. 65-81, 2007.

[49] R. Dantzer, J. C. O’Connor, G. G. Freund, R. W. Johnson, and K. W. Kelley, "From inflammation to sickness and depression: when the immune system subjugates the brain," Nature Reviews Neuroscience, vol. 9, no. 1, pp. 46-56, 2008.

[50] R. Dantzer, "Cytokine-induced sickness behavior: where do we stand?" Brain, Behavior, and Immunity, vol. 15, no. 1, pp. 7-24, 2001.

[51] A. H. J. van den Biggelaar, J. Gussekloo, A. J. M. de Craen et al., "Inflammation and interleukin-1 signaling network contribute to depressive symptoms but not cognitive decline in old age," Experimental Gerontology, vol. 42, no. 7, pp. 693-701, 2007.

[52] M. A. Kling, S. Alesci, G. Csako et al., "Sustained low-grade pro-inflammatory state in unmedicated, remitted women with major depressive disorder as evidenced by elevated serum levels of the acute phase proteins C-reactive protein and serum amyloid A," Biological Psychiatry, vol. 62, no. 4, pp. 309-313, 2007.

[53] V. Vaccarino, B. D. Johnson, D. S. Sheps et al., "Depression, inflammation, and incident cardiovascular disease in women with suspected coronary ischemia: the National Heart, Lung, and Blood Institute-sponsored WISE study," Journal of the American College of Cardiology, vol. 50, no. 21, pp. 2044-2050, 2007.

[54] V. Michopoulos, S. D. Norrholm, and T. Jovanovic, "Diagnostic biomarkers for posttraumatic stress disorder: promising horizons from translational neuroscience research," Biological Psychiatry, vol. 78, no. 5, pp. 344-353, 2015.

[55] D. Baumeister, R. Akhtar, S. Ciufolini, C. M. Pariante, and V. Mondelli, "Childhood trauma and adulthood inflammation: a meta-analysis of peripheral C-reactive protein, interleukin-6 and tumour necrosis factor- $\alpha$," Molecular Psychiatry, vol. 21, no. 5, pp. 642-649, 2016.

[56] C. L. Raison, L. Capuron, and A. H. Miller, "Cytokines sing the blues: inflammation and the pathogenesis of depression," Trends in Immunology, vol. 27, no. 1, pp. 24-31, 2006.

[57] S. D. Pollak, "Mechanisms linking early experience and the emergence of emotions: illustrations from the study of maltreated children," Current Directions in Psychological Science, vol. 17, no. 6, pp. 370-375, 2008.

[58] A. M. Shields, D. Cicchetti, and R. M. Ryan, “The development of emotional and behavioral self-regulation and social competence among maltreated school-age children," Development and Psychopathology, vol. 6, no. 1, pp. 57-75, 1994.

[59] M. A. Southam-Gerow and P. C. Kendall, "Emotion regulation and understanding: implications for child psychopathology and therapy," Clinical Psychology Review, vol. 22, no. 2, pp. 189-222, 2002.

[60] M. T. Tull, H. M. Barrett, E. S. McMillan, and L. Roemer, "A preliminary investigation of the relationship between emotion regulation difficulties and posttraumatic stress symptoms," Behavior Therapy, vol. 38, no. 3, pp. 303-313, 2007. 
[61] A. Powers, D. Cross, N. Fani, and B. Bradley, "PTSD, emotion dysregulation, and dissociative symptoms in a highly traumatized sample," Journal of Psychiatric Research, vol. 61, pp. 174179, 2015.

[62] C.-S. R. Li, C. Huang, P. Yan, Z. Bhagwagar, V. Milivojevic, and R. Sinha, "Neural correlates of impulse control during stop signal inhibition in cocaine-dependent men," Neuropsychopharmacology, vol. 33, no. 8, pp. 1798-1806, 2008.

[63] N. Owen, T. Poulton, F. C. Hay, V. Mohamed-Ali, and A. Steptoe, "Socioeconomic status, C-reactive protein, immune factors, and responses to acute mental stress," Brain, Behavior, and Immunity, vol. 17, no. 4, pp. 286-295, 2003.

[64] T. W. McDade, L. C. Hawkley, and J. T. Cacioppo, "Psychosocial and behavioral predictors of inflammation in middle-aged and older adults: the Chicago health, aging, and social relations study," Psychosomatic Medicine, vol. 68, no. 3, pp. 376-381, 2006.

[65] C. L. Raison, R. E. Rutherford, B. J. Woolwine et al., "A randomized controlled trial of the tumor necrosis factor antagonist infliximab for treatment-resistant depression: the role of baseline inflammatory biomarkers," JAMA Psychiatry, vol. 70, no. 1, pp. 31-41, 2013.

[66] J. D. Rosenblat, R. Kakar, M. Berk et al., "Anti-inflammatory agents in the treatment of bipolar depression: a systematic review and meta-analysis," Bipolar Disorders, vol. 18, no. 2, pp. 89-101, 2016. 

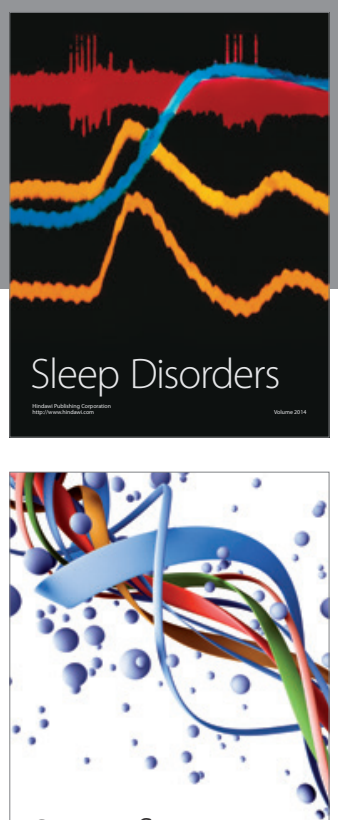

Scientifica
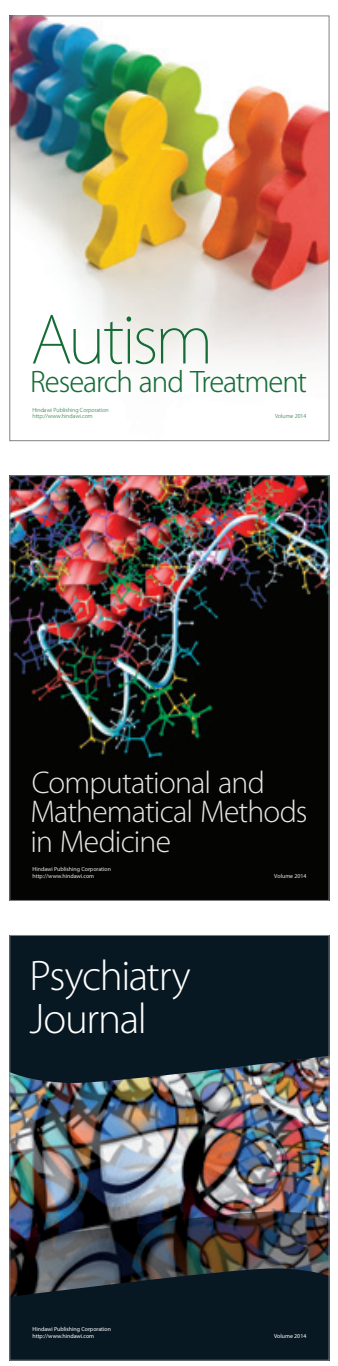
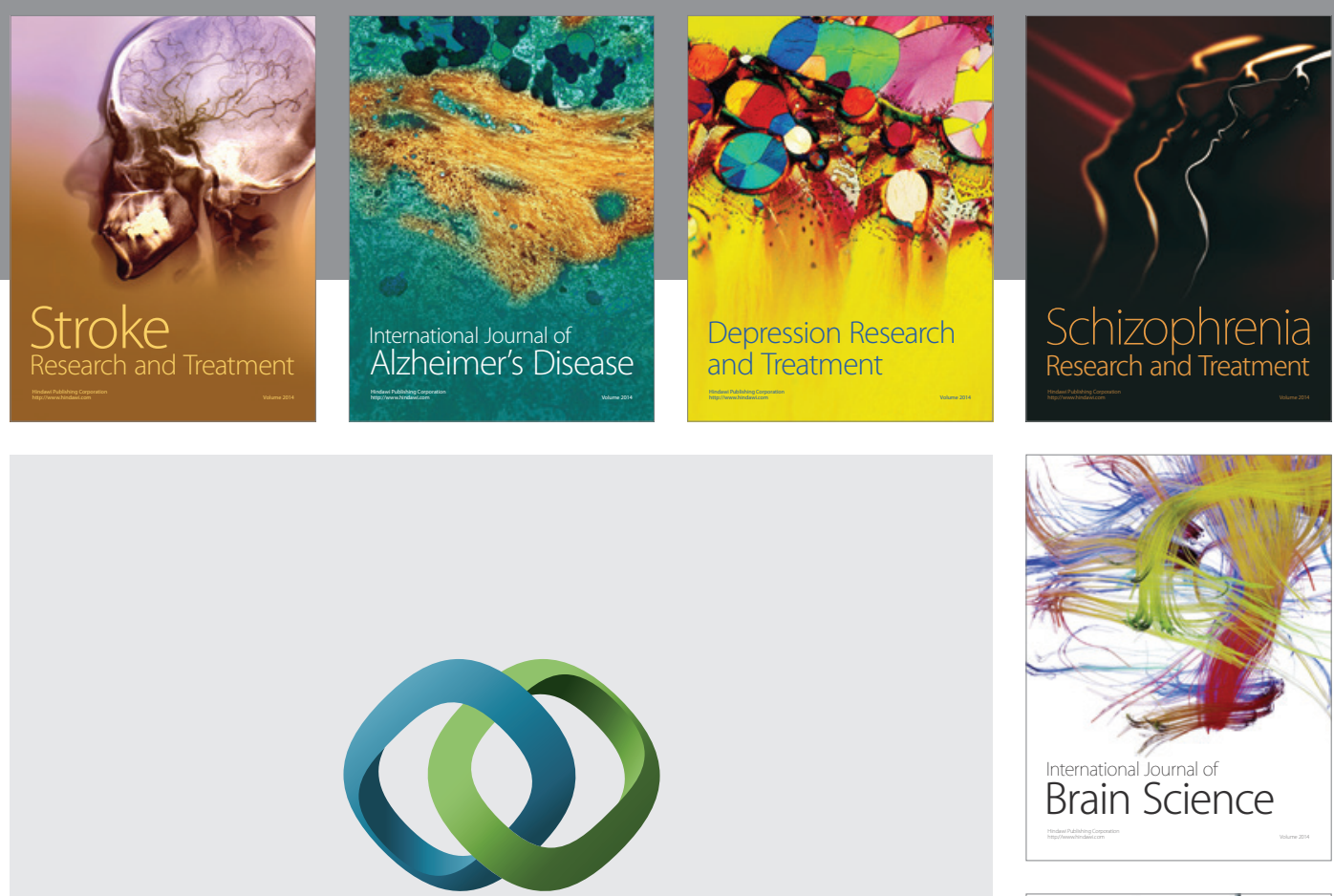

\section{Hindawi}

Submit your manuscripts at

http://www.hindawi.com
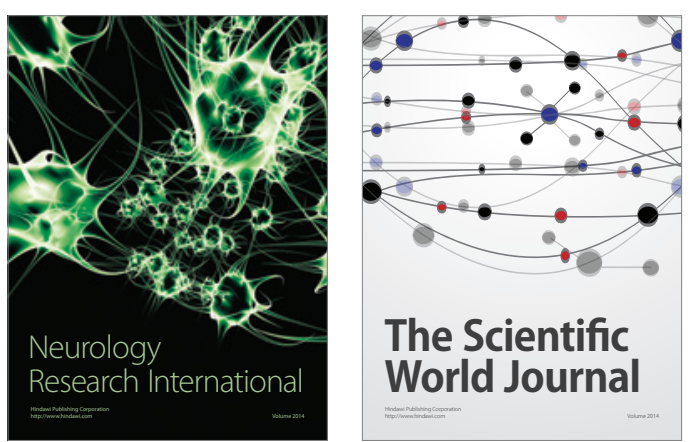

The Scientific World Journal

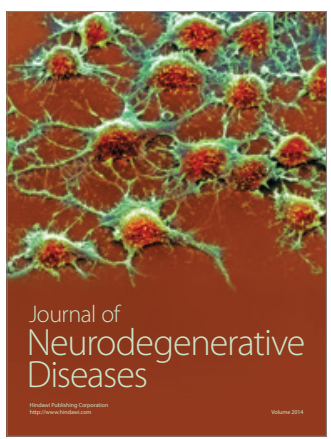

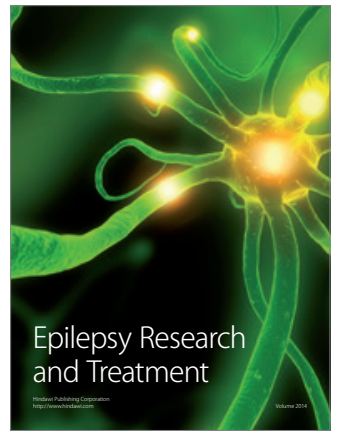

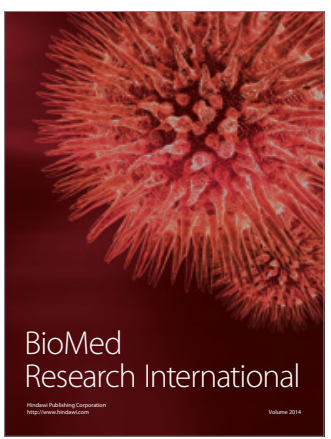

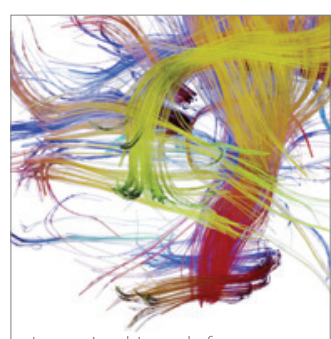

Brain Science

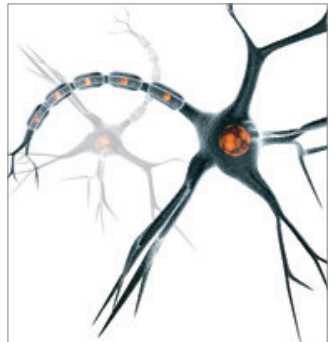

Neural Plasticity
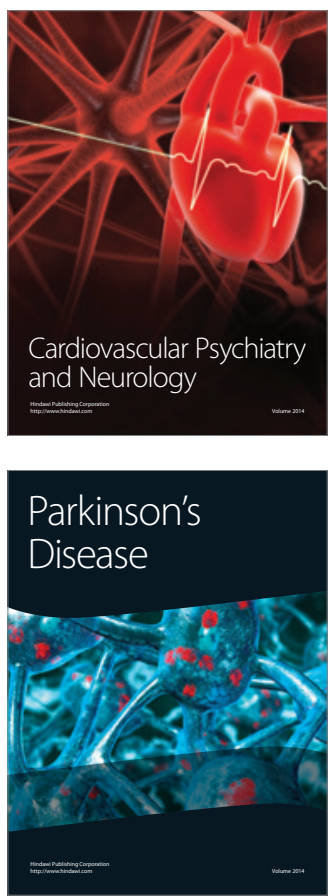